\title{
The Marine Natural Product Manzamine A Inhibits Cervical Cancer by Targeting the SIX1 Protein
}

\author{
Dev Karan,* Seema Dubey, Lucia Pirisi, Alexis Nagel, Ivett Pina, Yeun-Mun Choo, \\ and Mark T Hamann*
}

Cite This: J. Nat. Prod. 2020, 83, 286-295

Read Online

ABSTRACT: Natural products remain an important source of drug leads covering unique chemical space and providing significant therapeutic value for the control of cancer and infectious diseases resistant to current drugs. Here, we determined the antiproliferative activity of a natural product manzamine A (1) from an Indo-Pacific sponge following various in vitro cellular assays targeting cervical cancer (C33A, HeLa, SiHa, and CaSki). Our data demonstrated the antiproliferative effects of 1 at relatively low and non-cytotoxic concentrations (up to $4 \mu \mathrm{M}$ ). Mechanistic

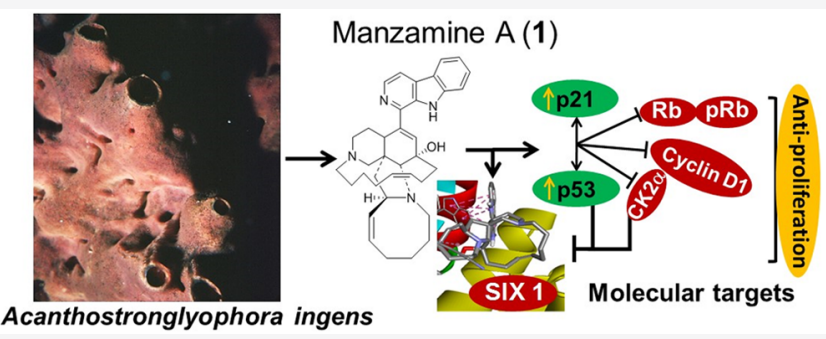
investigations confirmed that $\mathbf{1}$ blocked cell cycle progression in $\mathrm{SiHa}$ and CaSki cells at G1/S phase and regulated cell cycle-related genes, including restoration of p21 and p53 expression. In apoptotic assays, HeLa cells showed the highest sensitivity to 1 as compared to other cell types (C33A, SiHa, and CaSki). Interestingly, 1 decreased the levels of the oncoprotein SIX1, which is associated with oncogenesis in cervical cancer. To further investigate the structure-activity relationship among manzamine A (1) class with potential antiproliferative activity, molecular networking facilitated the efficient identification, dereplication, and assignment of structures from the manzamine class and revealed the significant potential in the design of optimized molecules for the treatment of cervical cancer. These data suggest that this sponge-derived natural product class warrants further attention regarding the design and development of novel manzamine analogues, which may be efficacious for preventive and therapeutic treatment of cancer. Additionally, this study reveals the significance of protecting fragile marine ecosystems from climate change-induced loss of species diversity.

$\mathrm{N}$ atural products have a long history of use in the treatment of various diseases including cancer. The primary sources of successful natural product cancer therapeutics have been from microbes and plants from the environment. In fact, more than $49 \%$ of currently used anticancer agents are either natural products or directly derived from natural products. ${ }^{1}$ Marine natural products from animals, plants, and bacteria continue to provide a highly productive resource for the discovery and development of new, innovative disease treatments with novel mechanisms of action. ${ }^{2}$ The marine environment is unique with regard to the high degree of competition among species, and the kinetics of metabolite transport in densely populated aqueous environments make marine organisms a rich source of structurally diverse and complex, bioactive natural products. ${ }^{3}$ Sessile marine organisms such as sponges are particularly rich in this regard, as they depend solely on either physical or chemical mechanisms to escape predation. Marine-derived bioactive terpenes alkaloids, macrolides, and other compounds isolated from aquatic fungi, cyanobacteria, algae, sponges, and tunicates, have all been found to exhibit various anticancer activities. ${ }^{4-7}$

Here, we studied the effect of a marine sponge product, namely, manzamine A (1), on cervical cancer cells. Cervical squamous dysplasia and cancer are due to infectious agents such as human papilloma virus (HPV), particularly the HPV types 16 and 18, which collectively account for an estimated $70 \%$ of all cervical cancer cases. For cervical cancer prevention, HPV vaccines (Gardasil, Gardasil 9, and Cervarix) protect against cervical infection with the HPV types included in the vaccines in women not previously exposed to HPV infection. ${ }^{8-10}$ However, HPV is a prophylactic vaccine, which must be introduced at a young age prior to HPV infection. Over $50 \%$ of women diagnosed with cervical cancer have stage II-IV disease and require combination of chemotherapy and radiation as their primary treatment. ${ }^{11}$ As proven clinically, these treatment modalities are associated with long-term side effects that significantly affect quality of life. Therefore, there is a continuous need to develop more effective therapies that can cure localized tumors and prevent progression and metastasis of cervical cancer.

Received: July 23, 2019

Published: February 5, 2020 


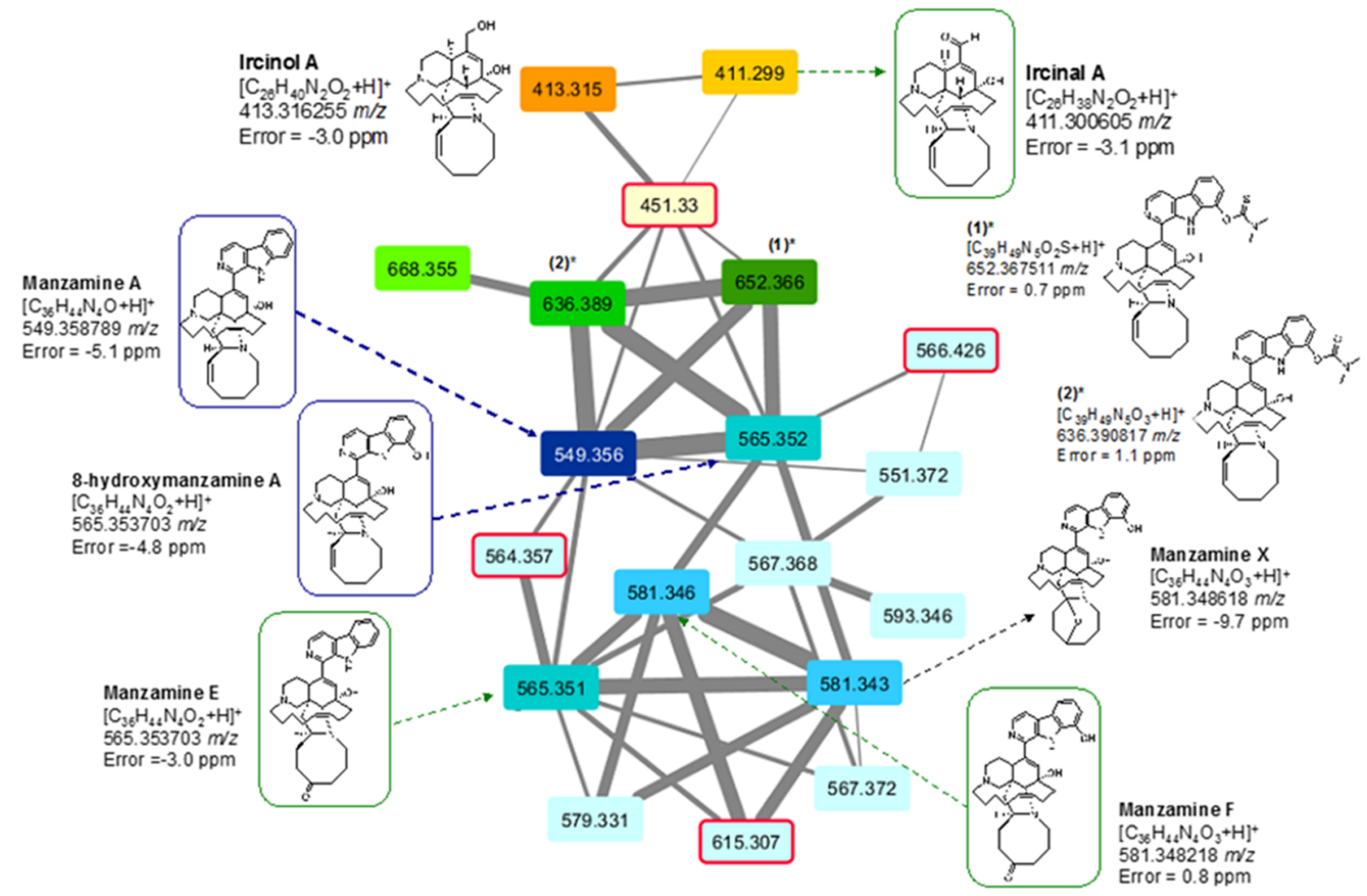

Figure 1. MolN analysis of sponge-derived alkaloids, with mass features color-coded by mass range (yellow $=400-500 \mathrm{~m} / z$; blue $=500-600 \mathrm{~m} / z$; green $=600-700 \mathrm{~m} / z$. The confirmed structures of $\mathbf{1}, \mathbf{3}, \mathbf{4}, \mathbf{5}$, and manzamine E were completed using ${ }^{1} \mathrm{H}$ NMR and MS/MS. The chemical structures of 1 and 4, the most abundant metabolites, were highlighted on blue boxes, while those of 3, 5, and manzamine E were on green boxes. *Two synthetic manzamine derivatives were used as control, compound 1, and compound 2, represented at the right of the figure. A full list of node retention times, exact and accurate mass measurements, and molecular formulas are represented in Supporting Information Table 1.

The manzamine alkaloids are isolated from Indo-Pacific sponges and represent a group of complex $\beta$-carboline alkaloids characterized by a unique nitrogen-containing polycyclic system. While some 80 manzamine analogues have been reported over the past two decades, it is likely that many more remain to be characterized and prepared through medicinal chemistry studies. This class of alkaloids has been previously reported to possess a wide variety of biological activity, and $\mathbf{1}$ has emerged as a promising drug candidate for the treatment of multiple disease conditions. ${ }^{12-14}$ However, studies examining the effect of 1 in cancer are very limited. In this study, we demonstrate that manzamine A (1) inhibits the growth of cervical cancer cells, inducing cell cycle arrest and stimulating apoptosis-related molecular pathways. We employed molecular networking (MolN) to facilitate the design of novel analogues that may harbor improved potential therapeutic activity against cervical cancer and other diseases. Furthermore, three novel manzamine analogues were discovered through mass spectrometry (MS)-based MolN, providing new directions for the investigation of manzamine A (1) and its mechanisms for the prevention or treatment of cervical cancer.

\section{RESULTS AND DISCUSSION}

Molecular Networking (MoIN) Facilitates Identification of Novel Manzamine A (1) Analogues. The discovery of natural product therapeutic leads continues to be greatly facilitated by highly sensitive mass spectrometry-based analysis that allows a higher-order view of metabolic diversity present within a biological sample. Posing a considerable challenge however is the streamlining of high-content MS data for identification of new mass species with potentially therapeutic properties. Here, we employed MoIN to aid in the discovery of new manzamine alkaloids based on comparisons between MS/ MS-derived data with available literature. Untargeted liquid chromatography (LC)-MS/MS analysis was performed on dried total alkaloid and fractionated extracts previously prepared from the Indonesian sponge Acanthostrongylophora sp. The presence of manzamine constituents manzamine A (1), manzamine D (2), manzamine F (3), 8-hydroxymanzamine A (4), ircinal A (5), and ircinol A (6) were previously detected by thin-layer chromatography and NMR. ${ }^{12,15}$ Samples were analyzed by LC-ESI-MS/MS (ESI = electrospray ionization) using an Impact II qTOF (Bruker) and spectral data networked using the GNPS open-source spectral networking script (Figure 1). ${ }^{16}$ Putative molecular formulas were generated for all features in 1 network (Supporting Information Table 1) based upon high-resolution (HRMS) data (not shown) and their relationship to known major and minor manzamine constituents (manzamine A $(\mathbf{1}),[\mathrm{M}+\mathrm{H}]^{+}$ $549.358550 \mathrm{~m} / z, \Delta=0.2 \mathrm{ppm}$; manzamine $\mathrm{E},[\mathrm{M}+\mathrm{H}]^{+}$ $565.353703 \mathrm{~m} / z, \Delta=0.1 \mathrm{ppm}$; manzamine $\mathrm{F}(3),[\mathrm{M}+\mathrm{H}]^{+}$ $581.348918 \mathrm{~m} / z, \Delta=-0.5 \mathrm{ppm})$, as well as ircinal A (5) [M $\left.+\mathrm{H}]^{+} 411.300598, \Delta=0.0 \mathrm{ppm}\right)$ and ircinol A $(6)\left([\mathrm{M}+\mathrm{H}]^{+}\right.$ $413.316214 \mathrm{~m} / z ; \Delta=0.1 \mathrm{ppm})$, which served as reference mass features. Fourteen mass features were assigned that, to our knowledge, do not correspond to any known, naturally occurring 1 derivative that could be determined through literature or database searching. 

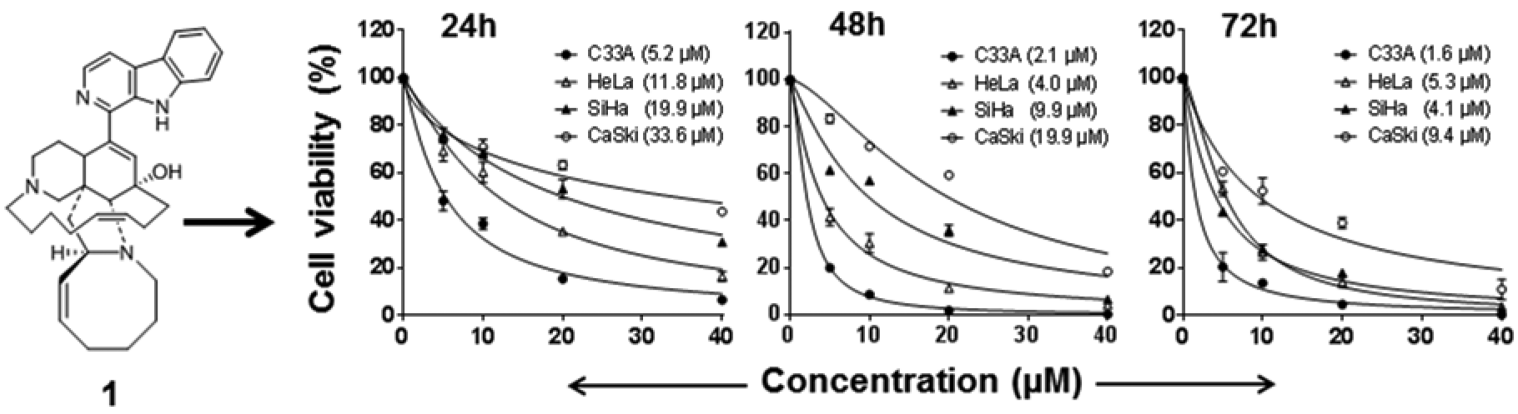

Figure 2. Biological effects of manzamine A (1) on cell viability of cervical cancer cell lines (C33A, HeLa, SiHa, and CaSki). Twenty thousand $(2 \times$ $\left.10^{4}\right)$ cells were seeded in 96-well plates and treated with a wide range from 1 to $40 \mu \mathrm{M}$ concentration of 1 for three time points $(24,48$, and $72 \mathrm{~h}$ ). The experiment was repeated at least three times in quadruplicate from different cell preparations with similar results. Percent viable cells were used to calculate $\mathrm{IC}_{50}$ values and are shown in the inset for all the cell lines at their respective time-points. The percent viability values represent mean \pm SEM.

\section{Biological Effect of Marine Sponge Natural Product Manzamine A (1) on Cervical Cancer Cell Growth. We} determined the biological activity of 1 on cervical cancer cells C33A (HPV-negative), HeLa, SiHa, and CaSki (HPV16/18positive) using a wide range of concentrations $(0-40 \mu \mathrm{M})$ at different time points $(24,48$, and $72 \mathrm{~h})$. The CellTiter-Glo cell viability assay determined the cell viability based on metabolically active cells producing adenosine triphosphate (ATP) in the cell culture. The cell viability assay showed that C33A and HeLa cells were more sensitive to (1) with the half maximal inhibitory concentration $\left(\mathrm{IC}_{50}\right)$ values of 2.1 and 4.0 $\mu \mathrm{M}$ at $48 \mathrm{~h}$ and values of 1.6 and $5.3 \mu \mathrm{M}$ at $72 \mathrm{~h}$ as compared to $\mathrm{SiHa}$ and CaSki cells. However, the CaSki cells were least sensitive to (1) with $\mathrm{IC}_{50}$ values of 19.9 and $9.4 \mu \mathrm{M}$ at 48 and 72 h, respectively (Figure 2 ).

To maintain the treatment uniformity across the cell lines, we selected optimal dose concentration from 1 to $4 \mu \mathrm{M}$ and treated C33A, HeLa, SiHa, and CaSki cell lines. The cellgrowth kinetics study showed that 1 significantly inhibited cervical cancer cell proliferation in a time- and dose-dependent manner as compared to vehicle dimethyl sulfoxide (DMSO) control. This antiproliferation cancer cell activity was further supported by the colony formation assays, where 2 and $4 \mu \mathrm{M}$ of manzamine A (1) inhibited the cell growth demonstrating a sustained, long-term ability of $\mathbf{1}$ to suppress cervical cancer cell growth (Supporting Information Figure 1).

On the basis of MoIN, the additional manzamine-related compounds such as manzamine D (2), manzamine F (3), 8hydroxymanzamine A (4), and ircinal A (5) were tested for their biological activities targeting cervical cancer cell lines (SiHa and CaSki). Manzamine D (2), 3, and 5 did not reveal any effect on cervical cancer cell growth; however, compound 4 showed inhibition of both $\mathrm{SiHa}$ and CaSki cells (Supporting Information Figure 2). Since 1 and 4 exhibited antiproliferation of cancer cells, we further tested the effect of these two analogues on normal human keratinocytes $(\mathrm{HKc})$. On the one hand, we observed that the cell-growth kinetics of the normal $\mathrm{HKc}$ treated with 8-hydroxymanzamine A (4) at $4 \mu \mathrm{M}$ concentration was inhibited significantly $(p=0.0022$ at $48 \mathrm{~h}$ and $p=0.0004$ at $72 \mathrm{~h}$ ) as compared to DMSO control. On the other hand, manzamine A (1) showed no adverse effect on normal HKc (Supporting Information Figure 3). These data suggest that compound $\mathbf{4}$ could be toxic to human normal cells, while 1 is non-cytotoxic.

Several groups have been pushing the boundaries of existing MolN applications to expand their utility for natural product dereplication. Notable efforts in this arena have been led by the lab of Crawford, who has employed MolN and bioinformaticsguided isotopic labeling to characterize elusive structural characteristics of the unstable and genotoxic E. coli-derived colibactin class that plays a role in colorectal cancer pathogenesis. ${ }^{17,18}$ However, despite its potential utility, only a small number of studies have exploited MolN as a tool to expedite the discovery and evaluation of new druglike smallmolecule natural products. Our networking results also support $\mathrm{MolN}$ as a valuable workflow component for the discovery of new manzamine lead molecules. MS-based MolN analysis facilitated the identification of putative manzamine A (1)related structures that would have been challenging to identify using only MS information. Interestingly, two of these compounds contain modifications at the $\beta$-carboline moiety, a chemical group that has previously been shown to be important to 1 bioactivity and mechanism of action. Manzamine A (1) cytotoxicity has been linked to $\beta$-carbolinemediated DNA intercalation in the major groove, contributing to cell-cycle arrest. ${ }^{19,20}$ While this group is known to stabilize associations with GSK $3 \beta$-binding, a similar binding mode may be approximated with alternative heterocycles, which retain desirable, selective kinase-inhibiting effects in the absence of DNA associations. ${ }^{21}$ Because of the differing routes of action of the $\beta$-carboline and ring systems, replacement of the $\beta$ carboline may offer a promising strategy for the development of a better lead molecule with activity against cancer cells.

Differential Activation of $\mathrm{G} 1$ Phase Arrest and Apoptosis by Manzamine A (1). To understand the mechanism of manzamine A (1)-mediated cell growth inhibition, we analyzed the cell-cycle distribution by flow cytometry in C33A, HeLa, SiHa, and CaSki cell lines. At $24 \mathrm{~h}$ of treatment, C33A and HeLa cells showed minimal differences in the cell-cycle distribution. However, there was a significant decrease in cell population in G0/G1 at 48 ( $p=$ $0.043)$ and $72(p=0.0012)$ hours with a corresponding increase in sub-G1 cell population as compared to DMSO control (Figure 3A). C33A cells also showed a significant decrease in $\mathrm{G} 2 / \mathrm{M}$ cell population at $48(p=0.005)$ and $72 \mathrm{~h}$ $(p=0.02)$ as compared to HeLa cells. On the contrary, compound $\mathbf{1}$ induces a significant increase in cell population at G0/G1 phase with a simultaneous decrease in $S$ and G2/M phases in SiHa and CaSki cells (Figure 3A). Particularly at 4 $\mu \mathrm{M}$ concentration, the percentage of $\mathrm{G} 0 / \mathrm{G} 1$ cell population in $\mathrm{SiHa}$ and CaSki increased to 1.2 -fold $(p=0.001)$ and 1.4 -fold 
(A) Cell cycle analysis
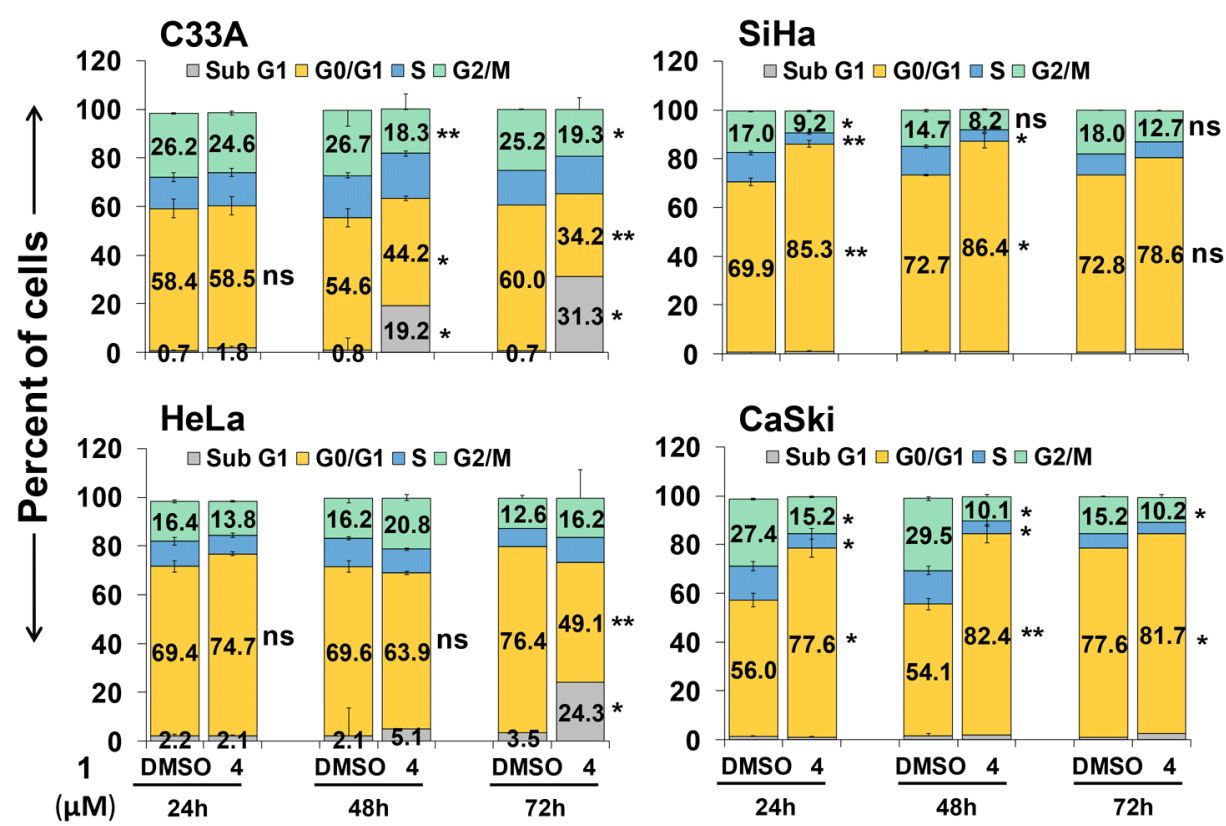

(B) Apoptotic assay

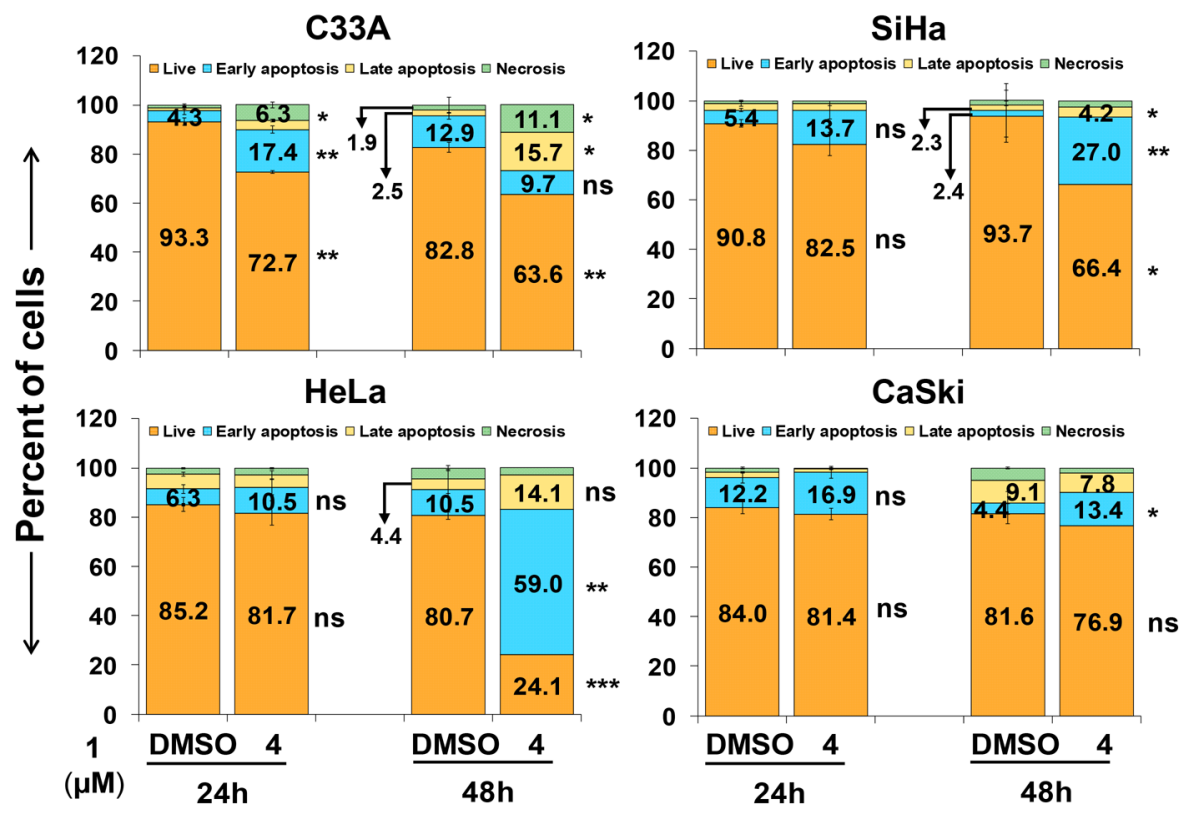

Figure 3. Representation of manzamine A (1) effect on cervical cancer cells treated with $4 \mu \mathrm{M}$ concentration. (A) Cell-cycle regulation at three different time points: cells were stained with PI, and analyzed by flow cytometry to estimate the amount of cells in each phase of the cell cycle; and (B) apoptosis at 24 and $48 \mathrm{~h}$ : cells were stained with Annexin-FITC and PI and analyzed by flow cytometry to estimate the amount of apoptosis and necrotic cell population. The values represent mean \pm SEM. DMSO serves as control. The significance level represents; $* p<0.05 ; * p<$ $0.001 ; * * *<0001$; and $\mathrm{ns}=$ nonsignificant.

$(p=0.011)$ at $24 \mathrm{~h}$, and 1.2 -fold $(p=0.03)$ and 1.5 -fold $(p=$ $0.006)$ at $48 \mathrm{~h}$ as compared to respective DMSO control.

Since programmed cell death (apoptosis) is a key mechanism by which anticancer drugs kill cancer cells, we determined the apoptotic effect of $\mathbf{1}$ on cervical cancer cells using annexin $\mathrm{V}$ and propidium iodide (PI) staining. Fortyeight hours of treatment with compound 1 triggered significant increase in apoptosis in HeLa cells $(p=0.005)$ as compared to
C33A, SiHa, and CaSki cells (Figure 3B). However, C33A cells appear to be sensitive, as $\mathbf{1}$ induces apoptosis $(p=0.002)$ and necrosis $(p=0.03)$ at $24 \mathrm{~h}$ with a further increase in late apoptosis $(p=0.03)$ and necrosis $(p=0.04)$ at $48 \mathrm{~h}$. Given the sensitivity of $\mathrm{C} 33 \mathrm{~A}$ and HeLa cell lines to 1, an increase in apoptosis was expected. Overall, the percentage of induced apoptotic cells (apoptosis + necrosis) at $4 \mu \mathrm{M}$ was higher at 48 $\mathrm{h}$ as compared to respective DMSO control in C33A (36.5 vs 

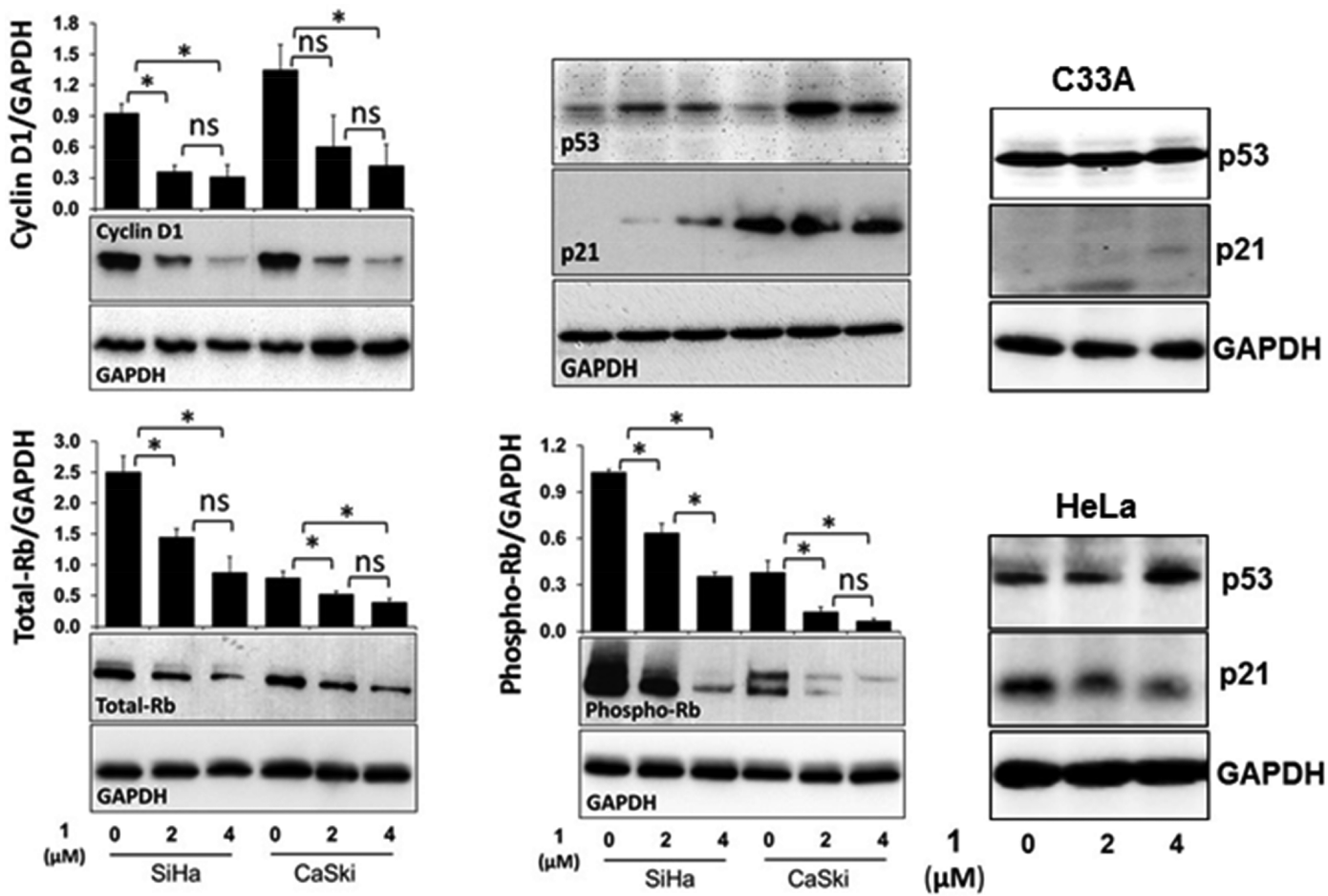

Figure 4. Effect of manzamine A (1) on the cell-cycle regulatory proteins in human cervical cancer cell lines. Following the overnight attachment of $1 \times 10^{6}$ cells/well, the cells were treated with manzamine A (1) at 2 and $4 \mu \mathrm{M}$ concentrations and incubated for $48 \mathrm{~h}$. Cell lysates were prepared for protein analysis.
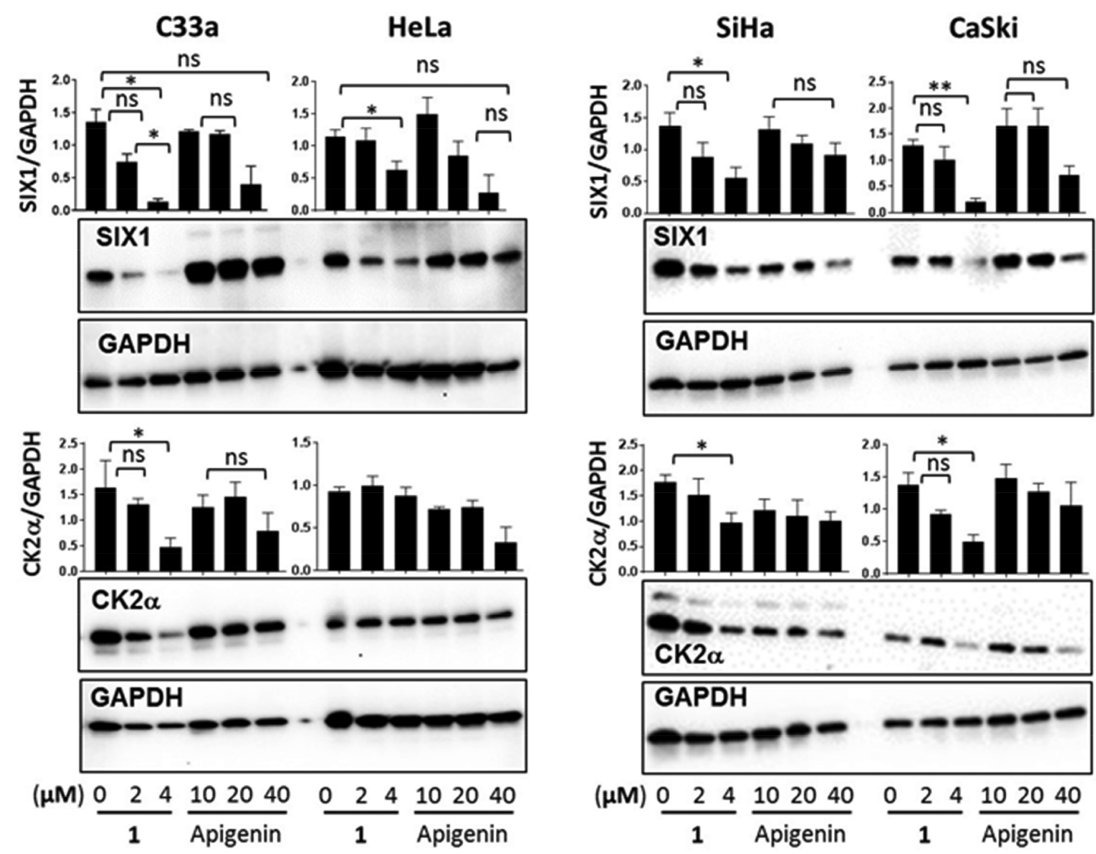

Figure 5. Analyzing the effect of manzamine A (1) and apigenin (a known inhibitor of CK2 $\alpha$ protein) on a homeodomain containing transcription factor SIX1 and CK2 $\alpha$ in human cervical cancer cell lines. Following the overnight attachment of $1 \times 10^{6}$ cells/well, the cells were treated with manzamine A (1) at 2 and $4 \mu \mathrm{M}$ or apigenin at 10, 20, and $40 \mu \mathrm{M}$ concentrations and incubated for $48 \mathrm{~h}$. Cell lysates were prepared for protein analysis.

17.3), HeLa (75.9 vs 19.3), SiHa (33.6 vs 6.4), and CaSki (23.2 vs 18.4), suggesting the role of 1 inducing apoptosis in cervical cancer cells. CaSki cells were the least sensitive and correspond to the $\mathrm{IC}_{50}$ values as in Figure 2.

Molecular Targets of Manzamine A (1). HeLa, SiHa, and CaSki cell lines represent the most common form of cervical cancer infected by the HPV type 16 ( $\mathrm{SiHa}$ and CaSki) and 18 (HeLa). Cervical cancer is primarily aggravated by the sustained expression of HPV-derived E6 and E7 oncogenes utilizing distinct mechanisms that affect cell-cycle checkpoints. For instance, E6 promotes p53 degradation, while E7 is associated with phosphorylation of retinoblastoma $(\mathrm{Rb})$ 


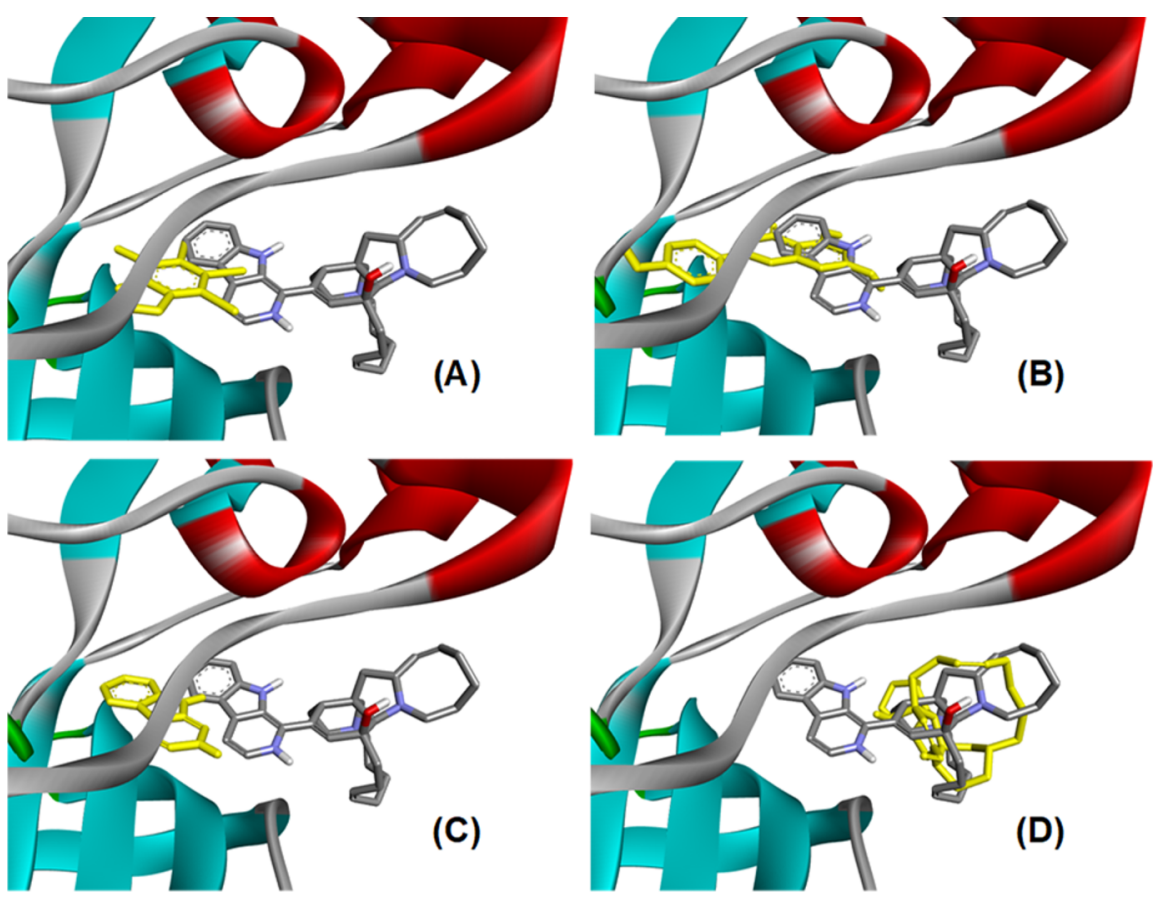

Figure 6. Comparison of CK2 $\alpha$ binding space for manzamine A (1) vs TBB (A), apigenin (B), $\beta$-carboline (C), and ircinal A (D). Manzamine A (1) structure is in gray color, while the yellow structures represent known inhibitors.

proteins. $^{22,23}$ Both $\mathrm{p} 53$ and $\mathrm{Rb}$ are tumor suppressor genes and play a critical role in maintaining cell-cycle regulation. Additionally, interaction of cyclin $\mathrm{D} 1$ with $\mathrm{Rb}$ protein and their expression levels correlate positively in association with tumorigenesis. Therefore, we sought to examine the molecular targets of 1 leading to anticancer activity in cervical cancer. Following treatment with the natural sponge product 1, we observed an increase in $\mathrm{p} 53$ and $\mathrm{p} 21$ protein levels and inhibition of cyclin $\mathrm{D} 1$, total $\mathrm{Rb}$, and phospho-Rb (Figure 4) in $\mathrm{SiHa}$ and CaSki cells, indicating that $\mathbf{1}$ may affect the regulation of both E6 and E7 oncogenes facilitating anticancer activities. HeLa cells showed a marginal increase in p53 level, while the level of p21 remains unchanged. On the contrary, HPV-negative C33A cells did not show any change in p53 protein level, while the $\mathrm{p} 21$ protein level was not detectable following 1 treatment. These data indicate a difference in mechanism of 1 in HPV-positive and HPV-negative cell lines. However, detailed mechanistic studies of $\mathbf{1}$ determining anticancer effect are required to further substantiate these observations.

Anticancer activity of sponge product $\mathbf{1}$ was further evident from the inhibition of SIX1 protein, which is a homeodomaincontaining transcription factor expressed at high levels in a variety of cancers, including cervical, colon, prostate, and breast cancers. ${ }^{24-27}$ Overexpression of SIX1 leads to decreased expression of p53 as well as aggressive clinical behavior of cancers and poor outcomes. ${ }^{26-28}$ Interestingly, the E7 oncogene induces SIX1 expression in cervical intraepithelial neoplasia and cervical cancer cells and accumulation of cyclin D1 in tumor cells, resulting in tumorigenesis. ${ }^{29,30}$ Our study demonstrated that 1 significantly inhibits the SIX1 protein both in HPV-negative C33A cells and in HPV-positive HeLa, $\mathrm{SiHa}$, and CaSki cells (Figure 5). These observations suggest that $\mathbf{1}$ induced cytotoxicity in cervical cancer cells across this dose range is not solely mediated by perturbing the E6/7 axis. Another possible mechanism of $\mathbf{1}$ action is linked with the inhibition of glycogen synthase kinase (GSK)-3 $\beta$, a biological effect that is supported by structural activity studies. ${ }^{13,31} \mathrm{~A}$ recent study in colorectal cancer showed that compound $\mathbf{1}$ induced cell-cycle arrest in G0/G1 via inhibition of cyclindependent kinases and caspase-dependent apoptotic cell death. $^{32}$ Furthermore, in pancreatic cancer studies $\mathbf{1}$ has been shown to sensitize pancreatic adenocarcinoma cells toward TRAIL-induced apoptosis, decreasing cell dissociation and migration by inhibiting autophagy through prevention of autophagosome turnover. ${ }^{33,34}$ It is not currently clear, however, whether 1 affects GSK3 $\beta$ and tumor necrosis factor (TNF)dependent pathways in cervical cancer. While the differences in the mechanism of $\mathbf{1}$ action could be due to differential regulatory pathways in different cancer types, we continued our efforts to determine the mechanism of anticancer activity in cervical cancer.

Molecular Docking of Manzamine A (1). Molecular docking assay is an effective tool to perform a preliminary evaluation of possible bioactivity. Previous studies have indicated that, in addition to E7 oncogene, SIX1 expression is regulated by protein kinase CK2. ${ }^{29,35}$ SIX1 contains conserved CK2 sites at positions Thr-490, Ile-491, Trp-492 and Asp-493. Inhibition of CK2 results in the diminished phosphorylation of SIX1 and leads to a dose-dependent arrest at the G2/M boundary, suggesting SIX1 as a plausible target of CK2. A molecular docking study of 1 was performed on CK2 kinase alpha subunit $(\mathrm{CK} 2 \alpha)$ protein to evaluate the ligandprotein interactions and binding affinity (Figure 6). The binding affinity and interactions of manzamine A (1) to CK2 $\alpha$ was compared to that of $\beta$-carboline, ircinal A (5), 4,5,6,7tetrabromobenzimidazole (TBB), and apigenin. TBB and apigenin are inhibitors of $\mathrm{CK} 2 \alpha$, while $\beta$-carboline and ircinal A (5) represent the partial structures of manzamine A (1). The results showed 1 has a better binding affinity $(-8.1 \mathrm{KCal} / \mathrm{mol})$ than the known CK2 $\alpha$ inhibitor TBB $(-7.4 \mathrm{KCal} / \mathrm{mol})$. Both apigenin $(-9.1 \mathrm{KCal} / \mathrm{mol})$ and $\beta$-carboline $(-8.6 \mathrm{KCal} / \mathrm{mol})$ 

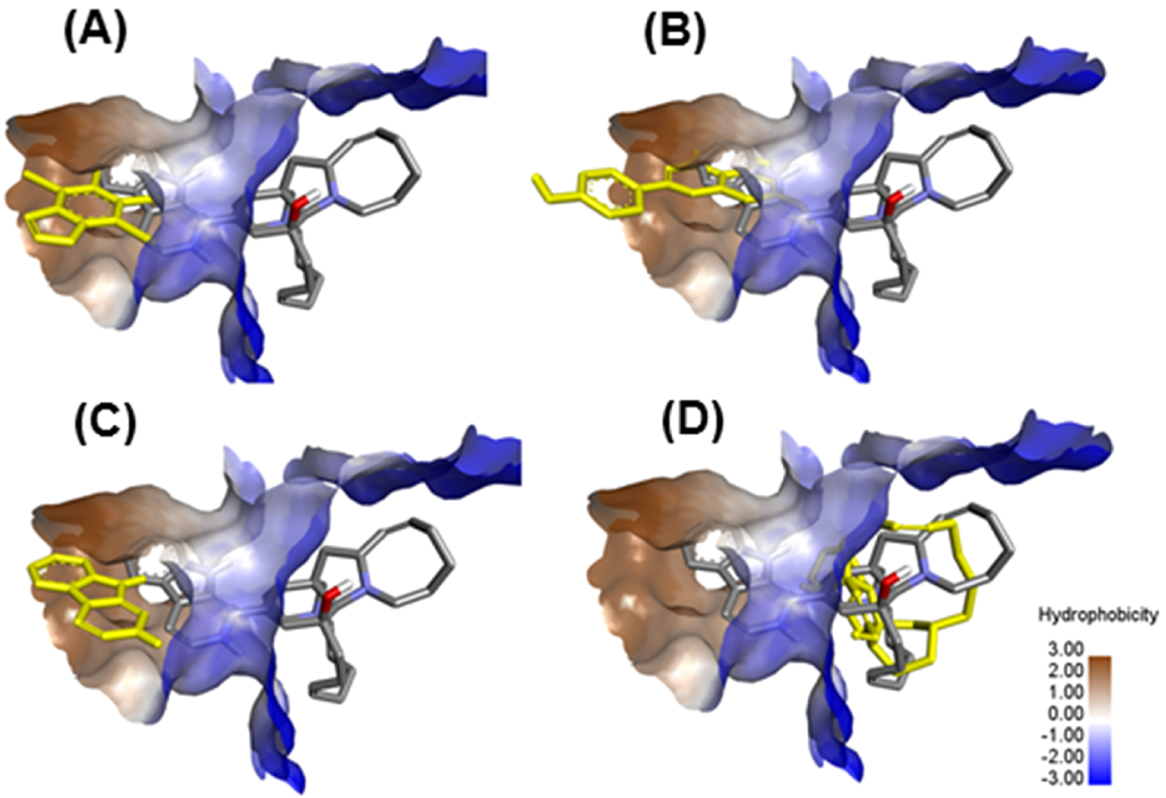

Figure 7. Comparison of $\mathrm{CK} 2 \alpha$ binding interactions for manzamine A (1) vs TBB (A), apigenin (B), $\beta$-carboline(C), and ircinal A (D). Manzamine A (1) structure is in gray color, while the yellow structures represent known inhibitors.

displayed better binding affinity than $\mathbf{1}$, while $5(-5.1 \mathrm{KCal} /$ mol) showed the most unfavorable binding affinity. The comparison of binding space and interactions between manzamine $\mathrm{A}(\mathbf{1})$ with $\mathrm{TBB}$, apigenin, $\beta$-carboline, and ircinal A (5) are shown in Figures 6 and 7. The results indicated that 1 shared similar binding interactions and space with the known CK $2 \alpha$ inhibitors, that is, TBB and apigenin. The displayed favorable binding of $\mathbf{1}$ to $\mathrm{CK} 2 \alpha$ is due to the strong hydrophobic interactions in the binding pocket (Figure 7). In support of our in silico docking model, we examined the effect of manzamine A (1) on CK2 $\alpha$ protein corresponding to apigenin effect on SIX1 protein level. We demonstrated that compound 1 decreased the level of $\mathrm{CK} 2 \alpha$ protein that corresponds to SIX1 in C33A, SiHa, and CaSki cells (Figure 5). Apigenin also decreased the proteins level of SIX1 and CK2 $\alpha$, however, at a much higher concertation $(40 \mu \mathrm{M})$. These data suggest that manzamine A (1) is almost 10 times more potent in inhibiting CK2 $\alpha$ and SIX1 target proteins as compared to the known inhibitor apigenin. Despite sensitivity of HeLa cells to 1, we did not observe CK $2 \alpha$ inhibition indicating an additional signaling mechanism of cancer cell killing. The study showed that $\beta$-carboline moiety of $\mathbf{1}$ is able to extend deep into the binding pocket to form hydrophobic interactions with similar amino acid residues (Met-163, Val116, Ile-66, Val-53, Val-45) as TBB and apigenin, while the ircinal moiety of $\mathbf{1}$ is located at the entrance of the binding pocket. Docking studies on $\beta$-carboline and ircinal A (5) are in agreement with 1 docking results, showing strong binding of $\beta$ carboline at the binding pocket, while $\mathbf{5}$ exhibits poor binding at the entrance of the binding pocket. These observations added another potential application of $\mathbf{1}$ as CK2 $\alpha$ inhibitor and present a new avenue of study for manzamine A (1).

Concluding Remarks. The manzamine class of marine alkaloids is attractive in its potential for the development of novel therapeutics. This group has been among the most extensively studied marine natural products currently, being found in more than 16 species of Indo-Pacific sponges distributed within distinct geographical regions. ${ }^{36}$ The first of this class, manzamine A (1), from Haliclona ${ }^{37}$ has demonstrated unprecedented potency against drug-resistant forms of the malaria parasite (Plasmodium spp.), ${ }^{38,39}$ showing greater potency and efficacy as compared to a number of Food and Drug Administration (FDA)-approved antimalarial drugs, including chloroquine. These molecules also show interesting activity as neuroprotective and anti-inflammatory agents, ${ }^{12-14}$ HIV/AIDS-associated opportunistic infections, ${ }^{12}$ and inhibition of cancer proliferative and metastatic activity. ${ }^{40} \mathrm{We}$ showed that manzamine A (1) treatment leads to significant decrease in the level of SIX1 and CK2 $\alpha$ proteins along with the regulation of cell-cycle related checkpoint proteins in cervical cancer cell lines suggesting that $\mathbf{1}$ might be active in vivo against cancers that express high levels of SIX1. In a direct comparison to apigenin, an inhibitor of $\mathrm{CK} 2 \alpha$, this study demonstrated that 1 is $\sim 10$ times more potent in inhibiting CK $2 \alpha$ and SIX1 proteins than apigenin. We have determined the oral toxicity profile of $\mathbf{1}$ in a healthy rodent (rat) model (unpublished) with a median lethal dose $\left(\mathrm{LD}_{50}\right)$ between 200 and $300 \mathrm{mg} / \mathrm{kg}$ body weight, comparable to that of acetaminophen (338 mg/kg orally) in mice, while observed weight loss at high doses resulted in termination of $\mathbf{1}$ for drug development by the Medicines for Malaria Venture, whose primary target patient group is very young children. It is anticipated that short-term weight loss would be less problematic or would not necessarily offset the therapeutic gain in cancer patients.

While advantageous for natural product dereplication in its current form, the MolN platform may benefit from the addition of applications that marry existing networking capabilities with utilities for molecular formula prediction and structural analysis, taking advantage of advanced algorithms that simultaneously analyze human resources management system (HRMS) and MS information contained within the data output. Given that current workflows require data transfer and parallel analysis within multiple software applications, an integrated platform such as this would represent a powerful tool for streamlined and efficient 
prioritization and deconvolution of molecular features with druglike properties. Given the natural diversity of manzamine compounds, it is possible that many more manzamine structures remain to be characterized within this rich reservoir of sponge secondary metabolites in addition to the potential to generate more useful molecules using rational drug design.

\section{EXPERIMENTAL SECTION}

Cell Culture. Human cervical cancer cell lines C33A, HeLa, SiHa, and CaSki were purchased from the American Type Culture Collection (ATCC). Cells were grown in Dulbecco's Modified Eagle Medium (DMEM) supplemented with $10 \%$ fetal bovine serum, $2 \mathrm{mM}$ L-glutamine, $100 \mathrm{U} / \mathrm{ml}$ of penicillin-streptomycin, and maintained at $37{ }^{\circ} \mathrm{C}$ with $5 \% \mathrm{CO}_{2}$ in a humidified incubator.

Generation of Normal Human Keratinocytes (HKc). The generation and processing of normal $\mathrm{HKc}$ from deidentified foreskin tissue samples has previously been described in our studies ${ }^{41}$ and was implemented with the following modifications: Dispase was used in lieu of collagenase, and a trypsinization step was added to release the epidermal cells. Briefly, to generate normal HKc primary cultures, the foreskin tissue, free of subcutaneous tissue, was placed in 10\% Dispase in phosphate buffer saline (PBS) and incubated overnight at $37^{\circ} \mathrm{C}$. The next day, the epidermis was separated from the dermis and processed mechanically by shredding the tissue into smaller pieces that were then treated with Trypsin/ethylenediaminetetraacetic acid (EDTA) for $10 \mathrm{~min}$ at $37^{\circ} \mathrm{C}$. Following the addition of fetal bovine serum $(10 \%)$ to stop the trypsin action, cells were collected by centrifugation and plated in keratinocytes serum free medium (KSFM).

Extraction and Isolation of Manzamine A (1). Lipophilic alkaloid extracts were previously prepared from the Indonesian sponge Acanthostrongylophora (collected from Manado Bay, Northern Sulawesi, Indonesia, in 2003). Crude material was separated using vacuum liquid chromatography, and $\mathbf{1}$ was purified by crystallization. Purified 1 was then transformed into its hydrochloride salt and recrystallized to reach high purity (>99\%) and optimized aqueous solubility as described previously. ${ }^{12,15}$

Cell Viability Assay. Cell viability was measured by using Cell Titer-Glo Luminescent Cell Viability Assay Kit (Promega) according to the manufacturer's instructions. Briefly, $2 \times 10^{4}$ cells were seeded in 96-well plates. The next day, the cells were treated with different concentrations of manzamine A (1) $(0-40 \mu \mathrm{M})$ for 24,48 , and $72 \mathrm{~h}$. Percent viability was calculated using the formula: percent (\%) viability $=100 \times($ treatment $/$ control $)$. The $\mathrm{IC}_{50}$ value was calculated from the dose-responsive sigmoid curve generated from $\%$ viability using GrpaphPad Prism 7 software.

Cell-Growth Kinetics. Cervical cancer cells $\left(1 \times 10^{6}\right)$ or HKc $(2$ $\times 10^{5}$ ) were plated in $100 \mathrm{~mm}$ tissue culture Petri dish or six-well plates. Following overnight incubation, cells were treated with 2 or 4 $\mu \mathrm{M}$ concentrations of manzamine A (1) derivatives for different time points $(24,48$, and $72 \mathrm{~h})$. DMSO was used as control. The cell proliferation activity was determined by cell counting under the microscope with a hemocytometer using trypan blue staining.

Colony-Formation Assay. Cancer cell lines were seeded at a density of $1 \times 10^{3}$ cells $/ \mathrm{ml}$ in six-well plates. Following overnight incubation, the cells were treated with manzamine A (1) (2 and 4 $\mu \mathrm{M})$ and incubated in a tissue culture incubator at $37{ }^{\circ} \mathrm{C}$ with $5 \%$ $\mathrm{CO}_{2}$. After one week, the developed cell colonies were fixed with $4 \%$ paraformaldehyde, stained with $0.5 \%$ crystal violet, and were photographed.

Cell-Cycle and Apoptosis Analysis. Cervical cancer cells were seeded and treated with different concentrations of manzamine A (1) as above. Following trypsinization, one part of the cells was washed with PBS and fixed in chilled $70 \%$ ethanol on ice for $30 \mathrm{~min}$. Cells were treated with RNase A $(1 \mathrm{mg} / \mathrm{mL})$ at room temperature for 30 min and stained with PI $(20 \mu \mathrm{g} / \mathrm{mL})$. Cell-cycle distribution was analyzed by flow cytometry (Beckman Coulter Epics Elite, Beckman). A minimum of 10000 events was counted per sample, and the data were analyzed using CXP software (Beckman Coulter) for the proportions of cells in G0/G1, S, and G2/M phases of the cell cycle. The second part of the cells was washed twice with PBS and resuspended in the buffer containing Alexa Fluor conjugated-Annexin$\mathrm{V}$ and PI (Life Technologies). The double staining of the cells was analyzed by flow cytometric analysis. A total of 10000 cell events were collected to analyze the extent of early, late apoptosis, or necrosis from each sample with the percentages of bound annexin- $\mathrm{V}^{+}$/ $\mathrm{PI}^{-}$, annexin $\mathrm{V}^{+} / \mathrm{PI}^{+}$, and annexin $\mathrm{V}^{-} / \mathrm{PI}^{+}$, respectively.

Protein Expression Analysis. C33A, HeLa, SiHa, and CaSki cells were seeded at a density of $1 \times 10^{6}$ cells $/ 100 \mathrm{~mm}$ Petri dish. The next day, the cells were treated with 2 or $4 \mu \mathrm{M}$ concentrations of manzamine A (1) or with 10,20 , or $40 \mu \mathrm{M}$ concentrations with apigenin (Sigma) and incubated for $48 \mathrm{~h}$. At the given time point, the cells were harvested by trypsinization, washed with PBS, and lysed using radio-immunoprecipitation assay (RIPA) buffer containing a cocktail of protease inhibitors (Sigma) to extract protein. Following quantification by colorimetric assay (Bio-Rad), an equal amount of protein was loaded on to sodium dodecyl sulfate (SDS)polyacrylamide gel and transferred to poly(vinylidene difluoride) (PVDF) membrane, which was blocked in 5\% nonfat dry milk in trisbuffered saline with tween-20 (TBST) and incubated with primary antibody against p53, p21, Cyclin D1, SIX1, GAPDH (Cell signaling) or CK2 $\alpha$ (R\&D Systems) at a 1:500 dilution. For total CK2 $\alpha, \mathrm{Rb}$, and $\mathrm{pRb}$, the blocking and antibody dilutions were made in $3 \%$ bovine serum albumin (BSA). The membrane was washed with TBST and incubated with secondary IgG HRP conjugate at 1:2000 dilution. The specific band of the protein was visualized with chemiluminescence reagent exposed onto BioMax Film (Kodak) and ChemoDoc-it ${ }^{2}$ imager (UVP).

Molecular Networking. Alkaloid samples were resolubilized in $100 \%$ methanol and analyzed on an online LC-ESI-MSMS system comprised of an Agilent 1100 series pump/autosampler/diode array coupled to a Bruker Impact II QqTOF. Samples (500 ng) were separated on a tetramethylsilane (TMS)-end-capped C18 RPLC column (Kinetex $5 \mu \mathrm{M} 100 \AA, 250 \times 4.6 \mathrm{~mm}$ column) with a gradient of $10-90 \%$ acetonitrile $+0.1 \%$ formic acid over $30 \mathrm{~min}(1 \mathrm{~mL} / \mathrm{min})$. Spectra were acquired within the $150-2000 \mathrm{~m} / z$ mass range, and the top three signals $(300-1700 \mathrm{~m} / z)$ selected for $\mathrm{MS}^{2}$ fragmentation by collision induced dissociation within a $2.0 \mathrm{Da}$ window $(50 \mathrm{eV})$, allowing for dynamic exclusion after three observations (30 s). Raw data were uploaded to the open-source Spectral Networking script at http://gnps.ucsd.edu ${ }^{16}$ and filtered for features greater than or equal to $2.5^{3}$ counts. Spectra were merged employing parent mass and MS/ MS fragment ion tolerance threshold of 0.1 Da. Only features with a minimum of five observations were retained in the network. Edges were filtered for cosine scores above 0.60 , retaining nodes that appeared in each other's respective top 10 most similar nodes. ${ }^{42,43}$ The resulting network was visualized in Cytoscape 3.4.0. ${ }^{44}$

Nuclear Magnetic Resonance (NMR). The ${ }^{1} \mathrm{H}$ and ${ }^{13} \mathrm{C}$ NMR spectra were recorded on a Bruker Nanobay $400 \mathrm{MHz}(9.4 \mathrm{~T}$ ) spectrometer in pyridine- $d_{5}$. Chemical shifts are expressed in parts per million and were referenced to residual solvent peaks $(\delta \mathrm{H} \mathrm{7.22,} \mathrm{7.58,}$ and $8.74 ; \delta \mathrm{C} 123.9,135.9,150.4)$. Preparation-scale high-performance liquid chromatography (HPLC) separation was performed using Waters 4000 Delta Prep system.

Molecular Docking. The MM2 energy-minimized three-dimensional (3D) structure of ligands, that is, manzamine A (1), $\beta$ carboline, ircinal A (5), 4,5,6,7-tetrabromobenzimidazole (TBB), and apigenin were optimized using ChemBio3D Ultra version 12.0. The crystal structure of Zea mays CK2 kinase alpha subunit (CK2 $\alpha$ ) (PDB ID: 1J91) receptor protein was obtained from Protein Data Bank (www.rcsb.org). ${ }^{45}$ The ligands and receptor for molecular docking experiments were prepared using AutoDockTools version 1.5.6, in which the polar hydrogens were added to these structures. ${ }^{46,47}$ The grit box parameter was set to cover the binding pocket in the receptor, with $x$-dimension $=20 ; y$-dimension $=20 ; z$-dimension $=20 ; X$-center $=21.774 ; Y$-center $=8.022 ;$ and $Z$-center $=19.144$. The grit box spacing was set as $1.0 \AA$. The docking was performed using AutoDock Vina, and the binding affinity values were measured. The outputs were 
visualized and analyzed using BIOVIA Discovery Studio Visualizer version 17.2.0.

Statistical Analysis. All experiments were performed in duplicate and repeated three to four times with similar results. All values are expressed as mean \pm standard error of the mean (SEM). Statistical analysis was performed using GraphPad Prism software (GraphPad Software, Inc.). The value of $p<0.05$ was considered statistically significant.

\section{ASSOCIATED CONTENT}

\section{s Supporting Information}

The Supporting Information is available free of charge at https://pubs.acs.org/doi/10.1021/acs.jnatprod.9b00577.

General procedures and molecular networking for alkaloid extracts. Network node data for known and unknown mass features observed in the manzamine molecular network and anticancer effect of (1) (PDF)

\section{AUTHOR INFORMATION}

\section{Corresponding Authors}

Dev Karan - Department of Pathology, MCW Cancer Center and Prostate Cancer Center of Excellence, Medical College of Wisconsin, Milwaukee, Wisconsin, United States; 10 orcid.org/ 0000-0002-0905-0886; Phone: 414-955-5574;

Email: dkaran@mcw.edu

Mark T Hamann - Department of Drug Discovery and Biomedical Sciences, Medical University of South Carolina, Charleston, South Carolina, United States; Phone: 843-7926659; Email: hamannm@musc.edu

\section{Authors}

Seema Dubey - Department of Pathology, MCW Cancer Center and Prostate Cancer Center of Excellence, Medical College of Wisconsin, Milwaukee, Wisconsin, United States

Lucia Pirisi - Department of Pathology, Microbiology and Immunology, University of South Carolina School of Medicine, Columbia, South Carolina, United States

Alexis Nagel - Department of Drug Discovery and Biomedical Sciences, Medical University of South Carolina, Charleston, South Carolina, United States; (1) orcid.org/0000-0002-37984002

Ivett Pina - Department of Drug Discovery and Biomedical Sciences, Medical University of South Carolina, Charleston, South Carolina, United States

Yeun-Mun Choo - Department of Chemistry, University of Malaya, Kuala Lumpur, Malaysia

Complete contact information is available at:

https://pubs.acs.org/10.1021/acs.jnatprod.9b00577

\section{Notes}

The authors declare no competing financial interest.

\section{ACKNOWLEDGMENTS}

D.K. is supported by the grants from the National Cancer Institute (Nos. CA169453 and CA179733). M.T.H. is supported by the pilot research funding, Hollings Cancer Center's Support Grant P30 (No. CA138313) at the Medical Univ. of South Carolina. We thank Dr. A. Choudhury for his experimental assistance. This work was initiated at the University of South Carolina School of Medicine, Columbia, South Carolina.

\section{REFERENCES}

(1) Newman, D. J.; Cragg, G. M. J. J. Nat. Prod. 2016, 79, 629-661.

(2) Newman, D. J.; Cragg, G. M.; Snader, K. M. Nat. Prod. Rep. 2000, 17, 215-234.

(3) Faulkner, D. J. Nat. Prod. Rep. 2002, 19, 1-48.

(4) Cragg, G. M.; Newman, D. J. Biochim. Biophys. Acta, Gen. Subj. 2013, 1830, 3670-3695

(5) Nair, D. G.; Weiskirchen, R.; Al-Musharafi, S. K. Acta Pharmacol. Sin. 2015, 36, 158-170.

(6) Mioso, R.; Marante, F. J.; Bezerra, R. S.; Borges, F. V.; Santos, B. V.; Laguna, I. H. Molecules 2017, 22, 208.

(7) Alessandra Gammone, M.; Riccioni, G.; Galvano, F.; D’Orazio, N. Anti-Cancer Agents Med. Chem. 2016, 16, 1549-1557.

(8) Beachler, D. C.; Kreimer, A. R.; Schiffman, M.; Herrero, R.; Wacholder, S.; Rodriguez, A. C.; Lowy, D. R.; Porras, C.; Schiller, J. T.; Quint, W.; Jimenez, S.; Safaeian, M.; Struijk, L.; Schussler, J.; Hildesheim, A.; Gonzalez, P. J. Natl. Cancer Inst. 2016, 108, djv302.

(9) Kreimer, A. R.; Gonzalez, P.; Katki, H. A.; Porras, C.; Schiffman, M.; Rodriguez, A. C.; Solomon, D.; Jimenez, S.; Schiller, J. T.; Lowy, D. R.; van Doorn, L. J.; Struijk, L.; Quint, W.; Chen, S.; Wacholder, S.; Hildesheim, A.; Herrero, R. Lancet Oncol. 2011, 12, 862-870.

(10) Kjaer, S. K.; Sigurdsson, K.; Iversen, O. E.; Hernandez-Avila, M.; Wheeler, C. M.; Perez, G.; Brown, D. R.; Koutsky, L. A.; Tay, E. H.; Garcia, P.; Ault, K. A.; Garland, S. M.; Leodolter, S.; Olsson, S. E.; Tang, G. W.; Ferris, D. G.; Paavonen, J.; Lehtinen, M.; Steben, M.; Bosch, F. X.; Dillner, J.; Joura, E. A.; Majewski, S.; Munoz, N.; Myers, E. R.; Villa, L. L.; Taddeo, F. J.; Roberts, C.; Tadesse, A.; Bryan, J.; Maansson, R.; Lu, S.; Vuocolo, S.; Hesley, T. M.; Saah, A.; Barr, E.; Haupt, R. M. Cancer Prev. Res. 2009, 2, 868-878.

(11) Pfaendler, K. S.; Wenzel, L.; Mechanic, M. B.; Penner, K. R. Clin. Ther. 2015, 37, 39-48.

(12) Yousaf, M.; Hammond, N. L.; Peng, J.; Wahyuono, S.; McIntosh, K. A.; Charman, W. N.; Mayer, A. M.; Hamann, M. T. J. Med. Chem. 2004, 47, 3512-3517.

(13) Hamann, M.; Alonso, D.; Martin-Aparicio, E.; Fuertes, A.; Perez-Puerto, M. J.; Castro, A.; Morales, S.; Navarro, M. L.; Del Monte-Millan, M.; Medina, M.; Pennaka, H.; Balaiah, A.; Peng, J.; Cook, J.; Wahyuono, S.; Martinez, A. J. Nat. Prod. 2007, 70, 13971405.

(14) Peng, J.; Kudrimoti, S.; Prasanna, S.; Odde, S.; Doerksen, R. J.; Pennaka, H. K.; Choo, Y. M.; Rao, K. V.; Tekwani, B. L.; Madgula, V.; Khan, S. I.; Wang, B.; Mayer, A. M.; Jacob, M. R.; Tu, L. C.; Gertsch, J.; Hamann, M. T. J. Med. Chem. 2010, 53, 61-76.

(15) Rao, K. V.; Donia, M. S.; Peng, J.; Garcia-Palomero, E.; Alonso, D.; Martinez, A.; Medina, M.; Franzblau, S. G.; Tekwani, B. L.; Khan, S. I.; Wahyuono, S.; Willett, K. L.; Hamann, M. T. J. Nat. Prod. 2006, 69, 1034-1040.

(16) Bandeira, N.; Tsur, D.; Frank, A.; Pevzner, P. A. Proc. Natl. Acad. Sci. U. S. A. 2007, 104, 6140-6145.

(17) Vizcaino, M. I.; Crawford, J. M. Nat. Chem. 2015, 7, 411-417.

(18) Vizcaino, M. I.; Engel, P.; Trautman, E.; Crawford, J. M. J. Am. Chem. Soc. 2014, 136, 9244-9247.

(19) Tu, L. C.; Chou, C. K.; Chen, C. Y.; Chang, Y. T.; Shen, Y. C.; Yeh, S. F. Biochim. Biophys. Acta, Gen. Subj. 2004, 1672, 148-156.

(20) Wahba, A. E.; Peng, J.; Kudrimoti, S.; Tekwani, B. L.; Hamann, M. T. Bioorg. Med. Chem. 2009, 17, 7775-7782.

(21) Waters, A. L.; Hill, R. T.; Place, A. R.; Hamann, M. T. Curr. Opin. Biotechnol. 2010, 21, 780-786.

(22) Huh, K.; Zhou, X.; Hayakawa, H.; Cho, J. Y.; Libermann, T. A.; Jin, J.; Harper, J. W.; Munger, K. J. Virol. 2007, 81, 9737-9747.

(23) Talis, A. L.; Huibregtse, J. M.; Howley, P. M. J. Biol. Chem. 1998, 273, 6439-6445.

(24) Xu, H. W.; Pirisi, L.; Creek, K. E. Virology 2015, 474, 144-153.

(25) Wan, F.; Miao, X.; Quraishi, I.; Kennedy, V.; Creek, K. E.; Pirisi, L. Int. J. Cancer 2008, 123, 32-40.

(26) Reichenberger, K. J.; Coletta, R. D.; Schulte, A. P.; VarellaGarcia, M.; Ford, H. L. Cancer Res. 2005, 65, 2668-2675.

(27) Coletta, R. D.; Christensen, K.; Reichenberger, K. J.; Lamb, J.; Micomonaco, D.; Huang, L.; Wolf, D. M.; Muller-Tidow, C.; Golub, 
T. R.; Kawakami, K.; Ford, H. L. Proc. Natl. Acad. Sci. U. S. A. 2004, 101, 6478-6483.

(28) Towers, C. G.; Guarnieri, A. L.; Micalizzi, D. S.; Harrell, J. C.; Gillen, A. E.; Kim, J.; Wang, C. A.; Oliphant, M. U.; Drasin, D. J.; Guney, M. A.; Kabos, P.; Sartorius, C. A.; Tan, A. C.; Perou, C. M.; Espinosa, J. M.; Ford, H. L. Nat. Commun. 2015, 6, 10077.

(29) Liu, D.; Zhang, X. X.; Xi, B. X.; Wan, D. Y.; Li, L.; Zhou, J.; Wang, W.; Ma, D.; Wang, H.; Gao, Q. L. Int. J. Oncol. 2014, 45, $1232-1240$

(30) Yu, Y.; Davicioni, E.; Triche, T. J.; Merlino, G. Cancer Res. 2006, 66, 1982-1989.

(31) Miyashita, K.; Nakada, M.; Shakoori, A.; Ishigaki, Y.; Shimasaki, T.; Motoo, Y.; Kawakami, K.; Minamoto, T. Anti-Cancer Agents Med. Chem. 2009, 9, 1114-1122.

(32) Lin, L. C.; Kuo, T. T.; Chang, H. Y.; Liu, W. S.; Hsia, S. M.; Huang, T. C. Mar. Drugs 2018, 16, 252.

(33) Guzman, E. A.; Johnson, J. D.; Linley, P. A.; Gunasekera, S. E.; Wright, A. E. Invest. New Drugs 2011, 29, 777-785.

(34) Kallifatidis, G.; Hoepfner, D.; Jaeg, T.; Guzman, E. A.; Wright, A. E. Mar. Drugs 2013, 11, 3500-3516.

(35) Ford, H. L.; Landesman-Bollag, E.; Dacwag, C. S.; Stukenberg, P. T.; Pardee, A. B.; Seldin, D. C. J. Biol. Chem. 2000, 275, 2224522254.

(36) Radwan, M.; Hanora, A.; Khalifa, S.; Abou-El-Ela, S. H. Cell Cycle 2012, 11, 1765-1772.

(37) Sakai, R.; Higa, T.; et al. J. Am. Chem. Soc. 1986, 108, 64046405.

(38) Rao, K. V.; Santarsiero, B. D.; Mesecar, A. D.; Schinazi, R. F.; Tekwani, B. L.; Hamann, M. T. J. Nat. Prod. 2003, 66, 823-828.

(39) El Sayed, K. A.; Kelly, M.; Kara, U. A.; Ang, K. K.; Katsuyama, I.; Dunbar, D. C.; Khan, A. A.; Hamann, M. T. J. Am. Chem. Soc. 2001, 123, 1804-1808.

(40) Ridley, R. G.; Matile, H.; Jaquet, C.; Dorn, A.; Hofheinz, W.; Leupin, W.; Masciadri, R.; Theil, F. P.; Richter, W. F.; Girometta, M. A.; Guenzi, A.; Urwyler, H.; Gocke, E.; Potthast, J. M.; Csato, M.; Thomas, A.; Peters, W. Antimicrob. Agents Chemother. 1997, 41, 677686.

(41) Pirisi, L.; Yasumoto, S.; Feller, M.; Doniger, J.; DiPaolo, J. A. J. Virol. 1987, 61, 1061-1066.

(42) Watrous, J.; Roach, P.; Alexandrov, T.; Heath, B. S.; Yang, J. Y.; Kersten, R. D.; van der Voort, M.; Pogliano, K.; Gross, H.; Raaijmakers, J. M.; Moore, B. S.; Laskin, J.; Bandeira, N.; Dorrestein, P. C. Proc. Natl. Acad. Sci. U. S. A. 2012, 109, E17431752.

(43) Winnikoff, J. R.; Glukhov, E.; Watrous, J.; Dorrestein, P. C.; Gerwick, W. H. J. Antibiot. 2014, 67, 105-112.

(44) Balkovec, J. M.; Hughes, D. L.; Masurekar, P. S.; Sable, C. A.; Schwartz, R. E.; Singh, S. B. Nat. Prod. Rep. 2014, 31, 15-34.

(45) Battistutta, R.; De Moliner, E.; Sarno, S.; Zanotti, G.; Pinna, L. A. Protein Sci. 2001, 10, 2200-2206.

(46) Sanner, M. F. J. Mol. Graph Model 1999, 17, 57-61.

(47) Trott, O.; Olson, A. J. J. Comput. Chem. 2009, 31, 455-461. 